\title{
3D visualization and 3D printing in abnormal gastrointestinal system manifestations of situs ambiguus
}

\author{
Dior Etherton $^{1,2}$, Lisa Tee ${ }^{3}$, Carley Tillett ${ }^{4}$, Yin Hong Wong ${ }^{5}$, Chai Hong Yeong ${ }^{5}$, Zhonghua Sun ${ }^{6}$ \\ ${ }^{1}$ Discipline of Physics and Astronomy, ${ }^{2}$ Discipline of Computing, School of Engineering, Computing and Mathematical Sciences, Curtin University, \\ Perth, Western Australia, Australia; ${ }^{3}$ School of Pharmacy and Biomedical Sciences, ${ }^{4}$ Curtin Hub for Immersive Visualisation and eResearch (HIVE), \\ Curtin University, Perth, Western Australia, Australia; ${ }^{5}$ School of Medicine, Faculty of Health and Medical Sciences, Taylor's University, Subang \\ Jaya, Selangor, Malaysia; ${ }^{6}$ Discipline of Medical Radiation Sciences, School of Molecular and Life Sciences, Curtin University, Perth, Western \\ Australia, Australia
}

Correspondence to: Professor Zhonghua Sun. Discipline of Medical Radiation Sciences, School of Molecular and Life Sciences, Curtin University, Perth, WA 6845, Australia. Email: z.sun@curtin.edu.au.

Submitted May 14, 2020. Accepted for publication Jun 03, 2020.

doi: 10.21037/qims-20-661

View this article at: http://dx.doi.org/10.21037/qims-20-661

\section{Introduction}

Situs ambiguus is a rare congenital condition whereby sufferers have abnormally positioned viscera $(1,2)$. In such cases, it is dangerous to perform traditional diagnostic examinations, such as colonoscopy, thus anatomy is explored through invasive surgeries instead. By reconstructing a patient's viscera three-dimensionally, it is possible to trace the physical origins of the patient's symptoms, while eliminating the necessity for invasive surgery. We present a case of situs ambiguus with use of a combination of different approaches including $3 \mathrm{D}$ visualizations and patient-specific 3D printing technique to assist clinical diagnosis and patient management. This case highlights the potential value of utilising the latest visualization tools in the diagnostic assessment of complicated pathologies.

\section{Case study details}

\section{Clinical history and imaging examinations}

A 21-year-old female patient has presented with a history of abdominal pain, cyclical diarrhoea and constipation and waking at night since childhood. In the early years, the abdominal symptoms were thought to be associated with poor bowel movement. Pharmacological management with spasmolytic agents has not been beneficial. With increasing pain as the patient reaches adolescent, a series of imaging diagnoses including barium meal imaging, computed tomography (CT), ultrasound and magnetic resonance imaging (MRI) examinations were performed with diagnosis of heterotaxy syndrome with levocardia and prominent stomach malrotations.

Barium meal examination showed that the stomach lies in the right upper quadrant of the abdomen and is markedly elongated with the pylorus lying at approximately the L3 level, just to the right of midline (Figure 1A). There is evidence of malrotation with contrast exiting the pylorus and passing horizontally towards the left side of the abdomen (Figure 1B). The small bowel loops are located in the left side of the abdomen. Contrast medium is seen within the caecum which lies in the right lower quadrant, approximately two hours after the ingestion of barium. The small bowel loops are normal in appearance, demonstrating a normal faceted together configuration and normal mucosal appearance. In particular the terminal ileum is normal in appearance. No thick-walled, dilated or narrowed small bowel loops are seen. There is no evidence of mucosal ulceration or fistulous connection. MRI scan was performed to further confirm no features of inflammatory bowel disease.

CT and MRI examinations revealed that the spleen is presented as multiple splenunculi and are positioned within the right upper quadrant (Figure 2). The duodenum is positioned anteriorly as it passes to the left across the 

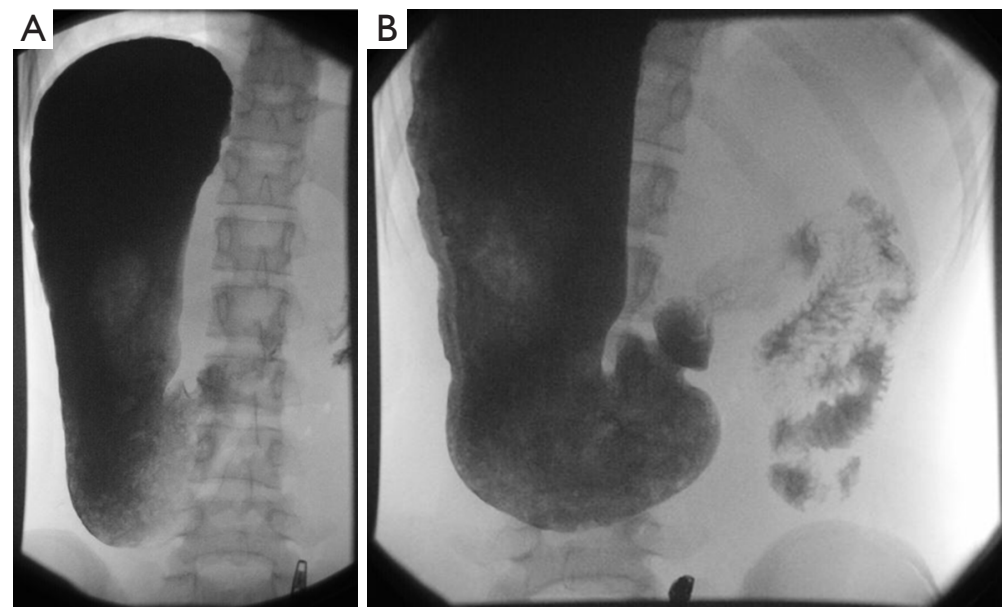

Figure 1 Barium meal examination showing stomach and duodenum position. (A) The stomach is entirely located in the right upper quadrant of the abdomen; (B) the duodenum and proximal part of jejunum are located in the left upper quadrant of the abdomen.
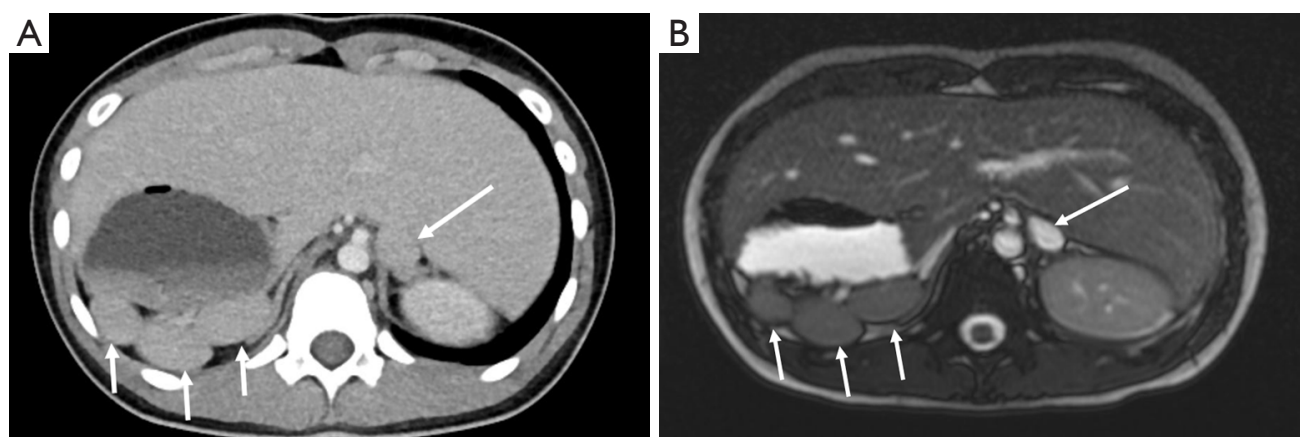

Figure 2 Three splenic segments located in the right abdomen. Contrast-enhanced CT (A) and 2D T2-weighted MRI images (B) shows iso-dense/iso-intense regions posterior to the stomach (short arrows) indicating the three separate splenic components. Long arrows refer to the inferior vena cava (IVC).

pancreas, but does not go retroperitoneally at all (Figure 3). The liver is midline but predominantly to the right abdomen (Figures 2,3). The gallbladder lies slightly to the left. The inferior vena cava (IVC) lies to the left of the aorta (Figure 2). The superior mesenteric vein (SMV) originally lies to the left of the superior mesenteric artery (SMA) but then passes to lie posteriorly and eventually to the right. There is a vein that courses 180 degrees around the SMA but this is not considered a tight twist. There is no oedema of the mesentery. The bowel is abnormal in terms of position with large intestine located in the right abdomen pushing small intestine to the left side of the abdomen (Figure 4). There is no free fluid or bowel wall thickening. Most of the pancreas appears to lie on the right. The appendix appeared to lie in the midline just above the bladder. The liver, gallbladder, pancreas, adrenals, kidneys and uterus are of normal appearance. No evidence of volvulus or bowel wall thickening.

The patient underwent diagnostic laparotomy for correction of malrotations with a focus on the pylorusduodenal malrotation. Based on the risk-benefit ratio, the decision was not to reverse the malrotation and an incidental laparoscopic appendectomy was performed.

The healthcare team for the patient includes the general practitioner, gastroenterologists, gynaecologist, nutritionist, and physiotherapist. Due to the complexity of the gastrointestinal malrotations, the gastroenterologist and gynaecologists are reluctant to offer surgical management. The proposed strategy is the management of pain through physiotherapy and heat pad which is complimented with 

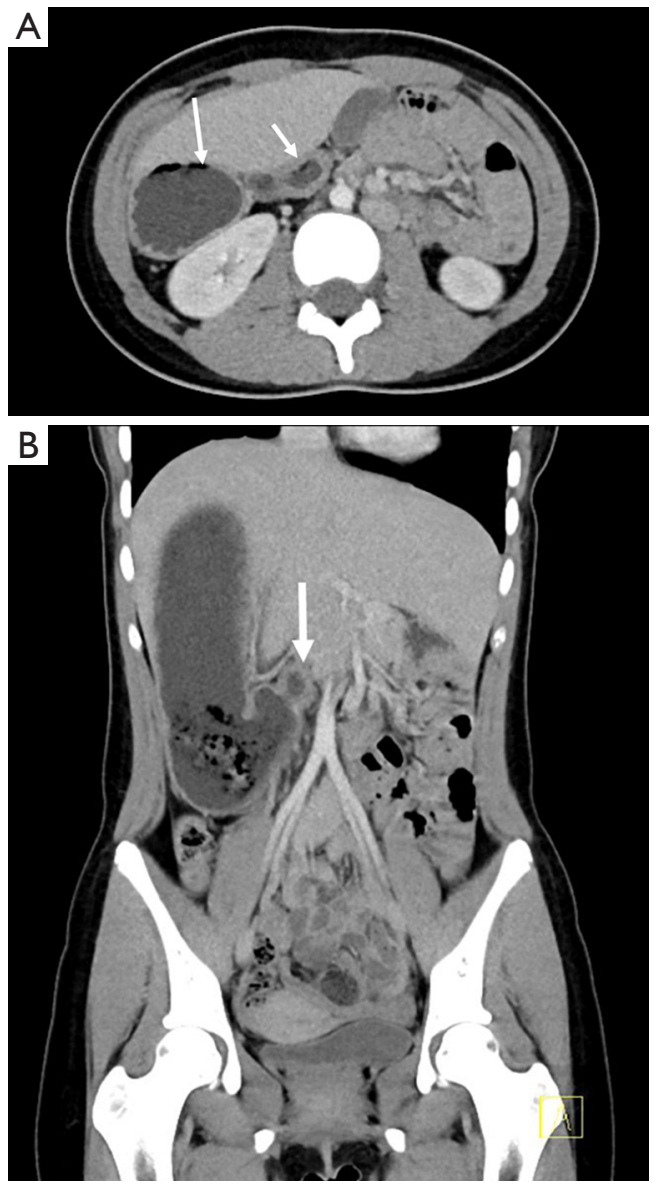

Figure 3 Contrast-enhanced CT images showing stomach and duodenum position. (A) 2D axial image shows that the stomach is located in the right abdomen posterior to the liver (long arrow) while duodenum (short arrow) extends anteriorly to the left abdomen; (B) coronal reformatted CT demonstrates the spatial relationship between stomach and duodenum (arrow).

pharmacological management using low dose amitriptyline and low dose oral contraceptive.

\section{Image post-processing and segmentation for 3D visualization and 3D printing}

Due to rarity of this case and complicated pathologies in the abdomen, CT and MRI images were used for $3 \mathrm{D}$ reconstructions and $3 \mathrm{D}$ printing of the abdominal organs. The segmentation of organs of interest was performed using Analyze 12.0 (AnalyzeDirect, Inc., Lexana, KS, USA), a commercial biomedical imaging software. By combining the semi-automatic and manual techniques provided in

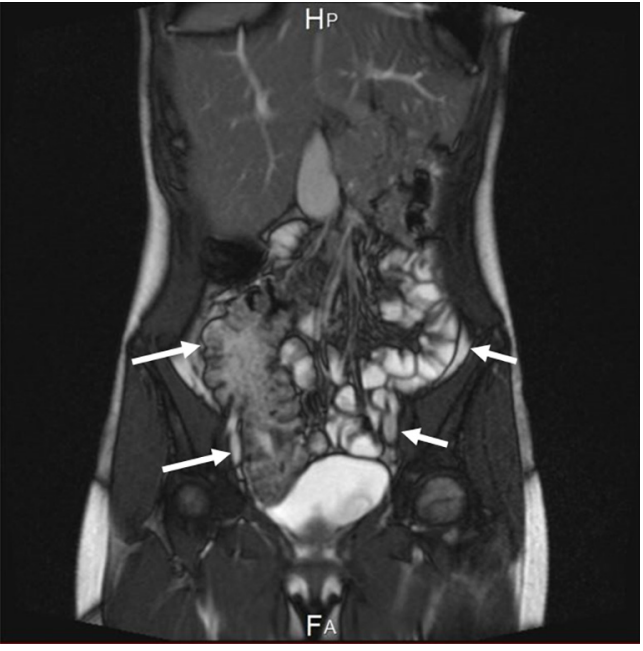

Figure 4 Coronal T2-weighted MR image shows abdominal organs with small intestine primarily in the left side of the abdomen (short arrows), while large intestine located in the right side of the abdomen (long arrows).

the Volume Edit window, a complete segmentation of the skeleton, kidneys, spleen, stomach, intestines, bladder, and uterus was obtained. The spleen, stomach, intestines, bladder, and uterus are abnormal in their positioning, and the skeleton and kidneys were segmented alongside them in order to provide a reference for further analysis (Figure 5). $3 \mathrm{D}$ reconstructed volumetric images were shown in a video demonstrating views from different angles (Video 1).

Once segmented, the organs were exported as Standard Tessellation Language (STL) files, which were sent to 3D printers for $3 \mathrm{D}$ printing. The STL files were imported into the Autodesk Mesh mixer (version 3.5.474, Autodesk Inc., San Rafael, CA, USA) for further edit. Deformities or freefloating objects were removed and any holes were fixed during the editing process. The bladder, kidney, stomach, spleen and uterus were further smoothened and created as hollow with a wall thickness of $1.5 \mathrm{~mm}$. The final STL files were printed with fused deposition modeling (FDM) printer using different thermoplastics. The skeleton model was printed with polylactic acid (PLA) on a Raise3D N2 Plus from Raise3D (Irvine, CA, USA). On the other hand, the bladder, kidney, stomach, spleen, and uterus models were printed on an Ultimaker 2 Extended+ from Ultimaker BV (Geldermalsen, Netherlands) using thermoplastic polyurethane (TPU) with shore hardness of 95A (Figure 6). The skeleton model took $\sim 247 \mathrm{~h}$ to complete with an estimated cost of USD 55. On the other hand, the estimated 

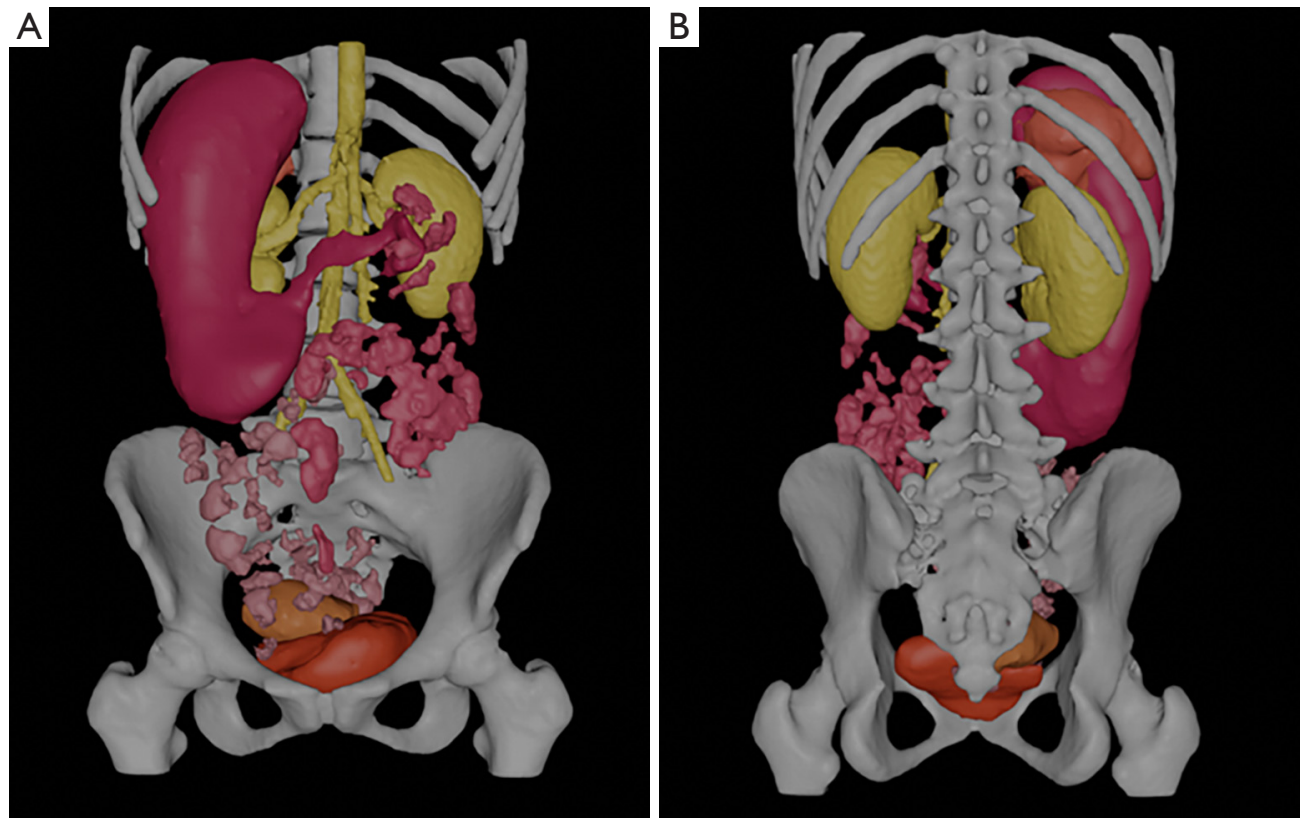

Figure $53 \mathrm{D}$ volume visualization of segmented data demonstrating these abdominal organs. (A) Frontal view; (B) posterior view. These organs were clearly segmented except for small and large intestine loops, which are discontinuous.
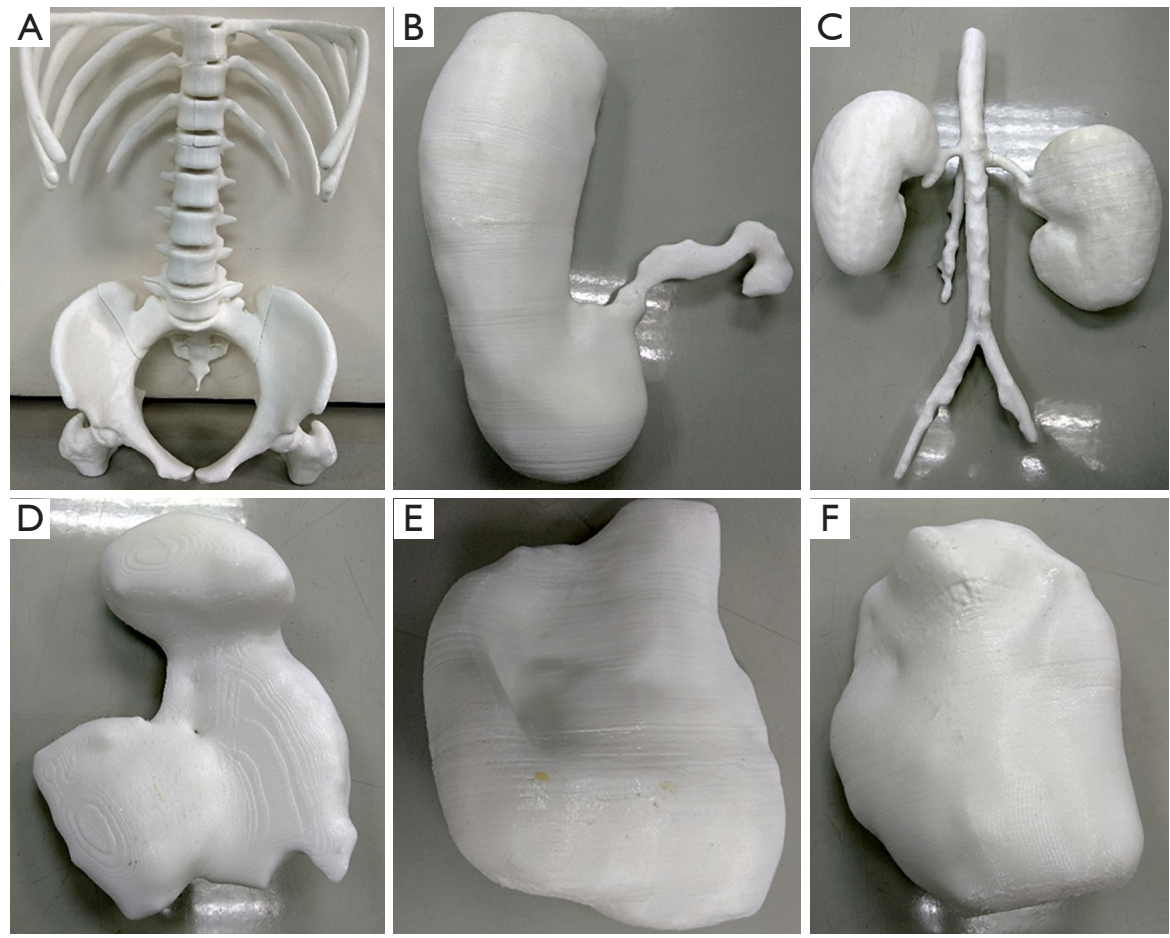

Figure 6 3D printed models of abdominal organs except for the small and large intestines. 3D printed skeleton (A), stomach (B), kidneys and abdominal aorta (C), spleen (D), bladder (E) and uterus (F) models. 

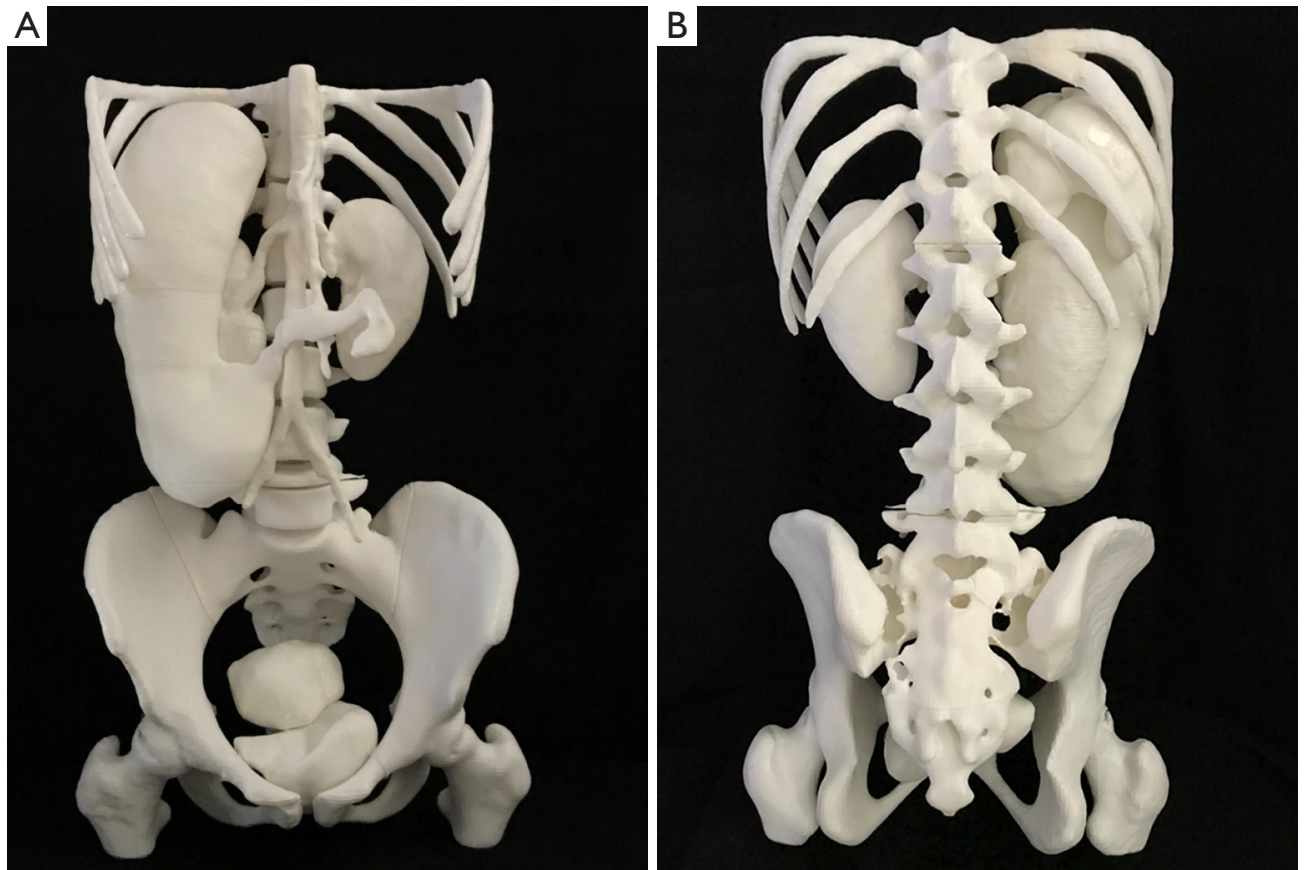

Figure 7 Demonstration of 3D printed models of abdominal organs after being assembled together. (A) Anterior view of the 3D printed models; (B) posterior view of the 3D printed models. Please note that the models were printed 1.5 times of the normal dimension of these organs.

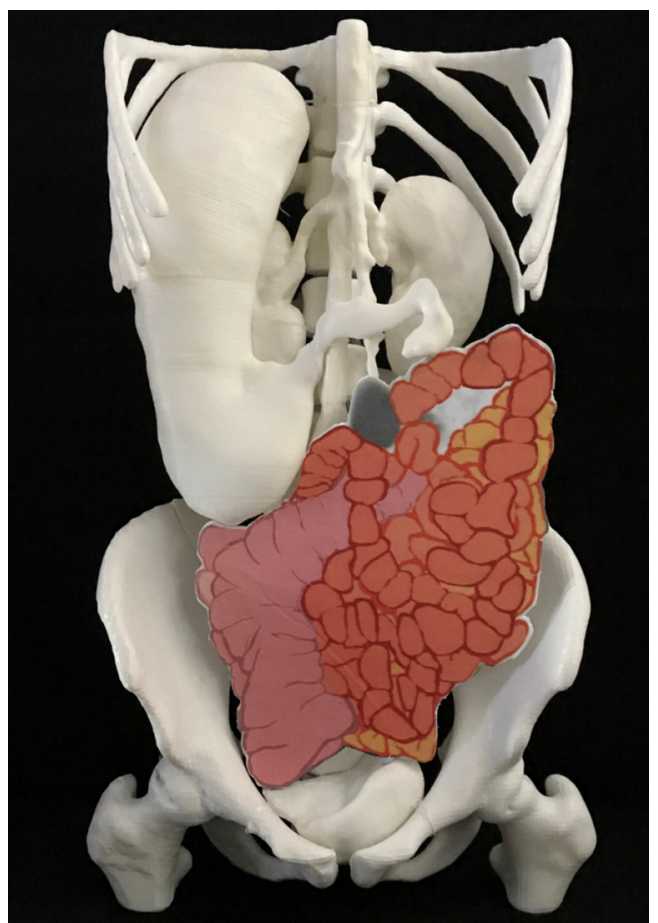

Figure $83 \mathrm{D}$ printed models with manual drawing of the intestines added to the missing part of the abdominal organs. printing time and cost for the bladder, kidney, stomach, spleen, and uterus were $\sim 250 \mathrm{~h}$ and USD 75 , respectively. After printing, individual printed organs were assembled together (Figure 7). Due to difficulty in segmenting small and large intestine loops on CT or MRI images, they could not be printed eventually due to discontinuity on the segmented volume images (Figure 5). Manual drawing of small and large intestines according to their imaging appearances on MRI images was added to the image of $3 \mathrm{D}$ printed models (Figure 8).

The segmented organs were assembled in a $3 \mathrm{D}$ software, Blender v 2.79b (Stitching Blender Foundation, The Netherlands), an open source software for further editing to form an entire body. This assembled model was used both to produce a stereoscopic video, and format suitable for display on a HoloTable. Short videos were generated to demonstrate the stereoscopic visual effect of Hologram (Video 2).

\section{Discussion}

This case study presents our experience of using a combination of $2 \mathrm{D}$ and $3 \mathrm{D}$ image visualizations in the diagnostic assessment of a rare disease, situs ambiguus. 
Although CT and MRI are commonly used imaging modalities in clinical practice with capability of producing $3 \mathrm{D}$ visualisations, they are still limited to providing images on $2 \mathrm{D}$ views. The lack of realistic $3 \mathrm{D}$ information by $\mathrm{CT}$ or MRI is overcome with use of advanced technologies such as $3 \mathrm{D}$ printing and Hologram, which are shown in this report. $3 \mathrm{D}$ printed physical models based on medical imaging data are increasingly used in medicine (3-12), while Hologram represents another innovative visualization tool showing applications in medical domain (13-16).

The hologram, 3D model and stereoscopic video yield an unmatched understanding of the abnormal organ positioning in three dimensions. When viewing the anatomy through CT scans and MRI images, the viewer is forced to project a series of $2 \mathrm{D}$ images into one $3 \mathrm{D}$ object. This is difficult to do without adequate experience, and also time consuming. Moreover, each time the healthcare team reconsiders the patient, they must study the CT and MRI images over again to reacquaint themselves with the anatomy. With this method of reconstructing the viscera of interest in $3 \mathrm{D}$, a spatial projection of the anatomy comes naturally, and each time it is revisited, the understanding is improved with reduction of viewing time.

By developing the $3 \mathrm{D}$ reconstructions in three different media, the ability to interpret the data is truly unparalleled. The stereoscopic video presents the data of interest concisely, and the hologram and model enable a viewer to focus on any part of the anatomy. The model is portable, making it a good tool to show to the various members of the patient's healthcare team, as well as the patient and their family, and serves as an educational tool for medical students.

\section{Acknowledgments}

Funding: This project is supported by Curtin HIVE Summer Internship Program (2019-2020).

\section{Footnote}

Conflicts of Interest: All authors have completed the ICMJE uniform disclosure form (available at http://dx.doi. org/10.21037/qims-20-661). ZS serves as an unpaid associate editor of Quantitative Imaging in Medicine and Surgery. The other authors have no conflicts of interest to declare.

Open Access Statement: This is an Open Access article distributed in accordance with the Creative Commons Attribution-NonCommercial-NoDerivs 4.0 International License (CC BY-NC-ND 4.0), which permits the noncommercial replication and distribution of the article with the strict proviso that no changes or edits are made and the original work is properly cited (including links to both the formal publication through the relevant DOI and the license). See: https://creativecommons.org/licenses/by-nc-nd/4.0/.

\section{References}

1. Fulcher AS, Turner MA. Abdominal manifestations of situs anomalies in adults. Radiographics 2002;22:1439-56.

2. Applegate KE, Goske MJ, Pierce G, Murphy D. Situs revisited: Imaging of the heterotaxy syndrome. Radiographics 1999;19:837-52.

3. Perica E, Sun Z. Patient-specific three-dimensional printing for pre-surgical planning in hepatocellular carcinoma treatment. Quant Imaging Med Surg 2017;7:668-77.

4. Sun Z, Lau I, Wong YH, Yeong CH. Personalized threedimensional printed models in congenital heart disease. J Clin Med 2019;8:522.

5. Sun Z, Liu D. A systematic review of clinical value of three-dimensional printing in renal disease. Quant Imaging Med Surg 2018;8:311-25.

6. Lau IWW, Liu D, Xu L, Fan Z, Sun Z. Clinical value of patient-specific three-dimensional printing of congenital heart disease: Quantitative and qualitative assessments. PLoS One 2018;13:e0194333.

7. Sindi R, Wong YH, Yeong CH, Sun Z. Development of patient-specific $3 \mathrm{D}$-printed breast phantom using silicone and peanut oils for magnetic resonance imaging. Quant Imaging Med Surg 2020;10:1237-48.

8. Witowski J, Wake N, Grochowska A, Sun Z, Budzyński A, Major P, Popiela TJ, Pędziwiatr M. Investigating accuracy of $3 \mathrm{~d}$ printed liver models with computed tomography. Quant Imaging Med Surg 2019;9:43-52.

9. Allan A, Kealley C, Squelch A, Wong YH, Yeong CH, Sun Z. Patient-specific 3D printed model of biliary ducts with congenital cyst. Quant Imaging Med Surg 2019;9:86-93.

10. Maier J, Weiherer M, Huber M, Palm C. Imitating human soft tissue on basis of a dual-material 3D print using a support-filled metamaterial to provide bimanual haptic for a hand surgery training system. Quant Imaging Med Surg 2019;9:30-42.

11. Sun Z, Jansen S. Personalized 3D printed coronary models in coronary stenting. Quant Imaging Med Surg 
2019;9:1356-67.

12. Valverde I, Gomez-Ciriza G, Hussain T, SuarezMejias C, Velasco-Forte MN, Byrne N, Ordoñez A, Gonzalez-Calle A, Anderson D, Hazekamp MG, Roest AAW, Rivas-Gonzalez J, Uribe S, El-Rassi I, Simpson J, Miller O, Ruiz E, Zabala I, Mendez A, Manso B, Gallego P, Prada F, Cantinotti M, Ait-Ali L, Merino C, Parry A, Poirier N, Greil G, Razavi R, Gomez-Cia T, Hosseinpour AR. Three dimensional printed models for surgical planning of complex congenital heart defects: an international multicenter study. Eur J Cardiothorac Surg 2017;52:1139-48.

13. Karmonik C, Elias SN, Zhang JY, Diaz O, Klucznik RP, Grossman RG, Britz GW. Augmented Reality with Virtual Cerebral Aneurysms: A Feasibility Study. World

Cite this article as: Etherton D, Tee L, Tillett C, Wong $\mathrm{YH}$, Yeong CH, Sun Z. 3D visualization and $3 \mathrm{D}$ printing in abnormal gastrointestinal system manifestations of situs ambiguus. Quant Imaging Med Surg 2020;10(9):1877-1883. doi: 10.21037/qims-20-661
Neurosurg 2018;119:e617-22.

14. Witowski J, Darocha S, Kownacki Ł, Pietrasik A, Pietura R, Banaszkiewicz M, Kamiński J, Biederman A, Torbicki A, Kurzyna M. Augmented reality and three-dimensional printing in percutaneous interventions on pulmonary arteries. Quant Imaging Med Surg 2019;9:23-9.

15. Jang J, Tschabrunn CM, Barkagan M, Anter E, Menze B, Nezafat R. Three-dimensional holographic visualization of high-resolution myocardial scar on HoloLens. PLoS One 2018;13:e0205188.

16. Bruckheimer E, Rotschild C, Dagan T, Amir G, Kaufman A, Gelman S, Birk E. Computer-generated real-time digital holography: first time use in clinical medical imaging. Eur Heart J Cardiovasc Imaging 2016;17:845-9. 